\title{
La craniectomía descompresiva como tratamiento del traumatismo encéfalo-craneano: Una visión actualizada
}

\section{Role of decompressive craniectomy in the management of severe traumatic brain Injury: An updated view}

\section{Juan Felipe Huidobro'}

${ }^{1}$ Neurocirujano Hospital Carlos Van Buren, Valparaíso. Servicio de Neurocirugía del Hospital Carlos Van Buren, Valparaíso, Chile. Cátedra de Neurocirugía, Universidad de Valparaíso, Chile.

\section{Resumen}

La hipertensión intracraneana es un importante indicador de mal pronóstico en los pacientes con traumatismo encéfalocraneano (TEC) severo. Pese a que la craniectomía descompresiva es una herramienta terapéutica probada y muy efectiva para disminuir la presión intracraneana, su rol en el manejo del TEC es controversial. Los beneficios de la craniectomía descompresiva sobre el desenlace neurológico de los pacientes no han sido respaldados por la evidencia; sus consecuencias sobre el proceso de la enfermedad traumática no son del todo claros y las múltiples complicaciones asociadas son difíciles de manejar. La estandarización de la terminología, un mejor entendimiento de los conceptos básicos que la fundamentan, de sus variantes técnicas, de sus efectos conocidos y de sus complicaciones, son esenciales para avanzar hacia una mejor comprensión de sus alcances y limitaciones, y así posiblemente aprovechar de mejor manera su potencial terapéutico.

Palabras clave: Craniectomía descompresión, traumatismo encéfalo-craneano.

\section{Abstract}

Intracranial hypertension is an important indicator of poor outcome in patients with severe traumatic brain injury. Although decompressive craniectomy has proven to be a highly effective therapy to decrease intracraneal pressure, its role in TBI management is controversial. The expected benefits of decompressive craniectomy on the patients neurological outcome have not been supported by the evidence; its impact on the pathophysiological cascade unleashed by the trauma are not well understood and the associated complications are hard to treat. Establishing a recognizable terminology, improving the comprehension of the basic principles, the surgical variations, the known effects and the complications, are essential for a better understanding of its reach and limitations, which may lead to a better use of its therapeutic potential.

Key words: Decompressive craniectomy, traumatic brain injury.

\section{Introducción}

El interés por prevenir, diagnosticar, monitorizar y tratar la hipertensión intracraneana (HTIC) en los pacientes con traumatismo encéfalo-craneano (TEC) severo, nació con la temprana observación de que la morbimortalidad de estos pacientes aumenta dramáticamente en los que evolucionan con una mayor carga de HTIC. Esta asociación, sería una consecuencia de dos procesos independientes. Por un lado, la compresión de estructuras neurovasculares que se produce durante los fenómenos de herniación cerebral y, por otro, el mayor riesgo de lesión cerebral secundaria (LCS) por hipoperfusión que tendrían los pacientes con HTIC (PPC = PAM-PIC) ${ }^{1}$.

\author{
Correspondencia a: \\ Juan Felipe Huidobro \\ San Ignacio 725, Valparaíso, Chile. \\ juanfehuidobro@gmail.com
}


A pesar de los múltiples avances en el ámbito del neurointensivo, el rol de la craniectomía descompresiva (CD) como tratamiento del TEC severo sigue siendo controversial.

En el presente artículo se analizan los conceptos, definiciones, efectos y evidencia más relevantes actualmente disponibles sobre $\mathrm{CD}$, para brindar una visión actualizada del problema, y así contribuir a destrabar los principales focos de controversia al respecto.

\section{Definiciones}

1. Craniectomía descompresiva: consiste en la remoción quirúrgica de una parte considerable de la bóveda craneana, asociada a una durotomía del área expuesta, con el propósito de aumentar el volumen del continente craneano, para disminuir la presión intracraneana (PIC) y/o aliviar la compresión mecánica secundaria al desplazamiento de estructuras ${ }^{2}$.

2. Craniectomía descompresiva primaria (CDP): es aquella $\mathrm{CD}$ que se realiza a un paciente en quien no se ha objetivado HTIC refractaria. Esto ocurre cuando el paciente debe ser sometido a una cirugía de cráneo de urgencia inmediata (por ejemplo para evacuar una lesión traumática con evidente efecto de masa) y las condiciones intraoperatorias impiden la reposición de la plaqueta ósea removida (por ejemplo por tumefacción cerebral), o el neurocirujano estima que el riesgo de evolución a HTIC es tan alto, que prefiere efectuar el procedimiento de manera profiláctica. En la actualidad, gracias a las mejoras en los sistemas de rescate prehospitalario y en el manejo anestésico y quirúrgico de los pacientes traumatizados, es infrecuente que las condiciones intraoperatorias obliguen al neurocirujano a realizar una CDP. Si a esto le agregamos el mayor acceso a la monitorización multimodal que tienen los pacientes en los centros de trauma, podemos entender por qué, en el contexto urbano del mundo desarrollado, el porcentaje de CDP ha disminuido significativamente en los últimos años, mientras su práctica se mantiene plenamente vigente en algunos escenarios especiales, como son los conflictos bélicos, y dentro de sistemas de salud menos desarrollados.

3. Craniectomía descompresiva secundaria (CDS): es aqueIla que se realiza a un paciente que está evolucionando con HTIC refractaria, es decir, que se encuentra bajo monitorización continua de PIC, recibiendo tratamiento en una unidad de cuidados intensivos, y que no tiene una lesión ocupante de espacio con indicación quirúrgica ${ }^{3}$.

La CDS es, por lo tanto, la que concita mayor interés desde la perspectiva del neurointensivo. Por qué, cuándo y cómo se debe realizar una CDS, son algunas de las principales controversias aún vigentes.

Diferenciar entre una CDP y una CDS es fundamental para interpretar correctamente la literatura médica correspondiente, entendiendo que, tanto la indicación como los resultados de la cirugía, deben ser analizados siempre considerando el contexto en el que se realiza la cirugía ${ }^{4}$. En la Figura 1, se presenta el algoritmo de toma de decisiones que se desprende de estas definiciones.

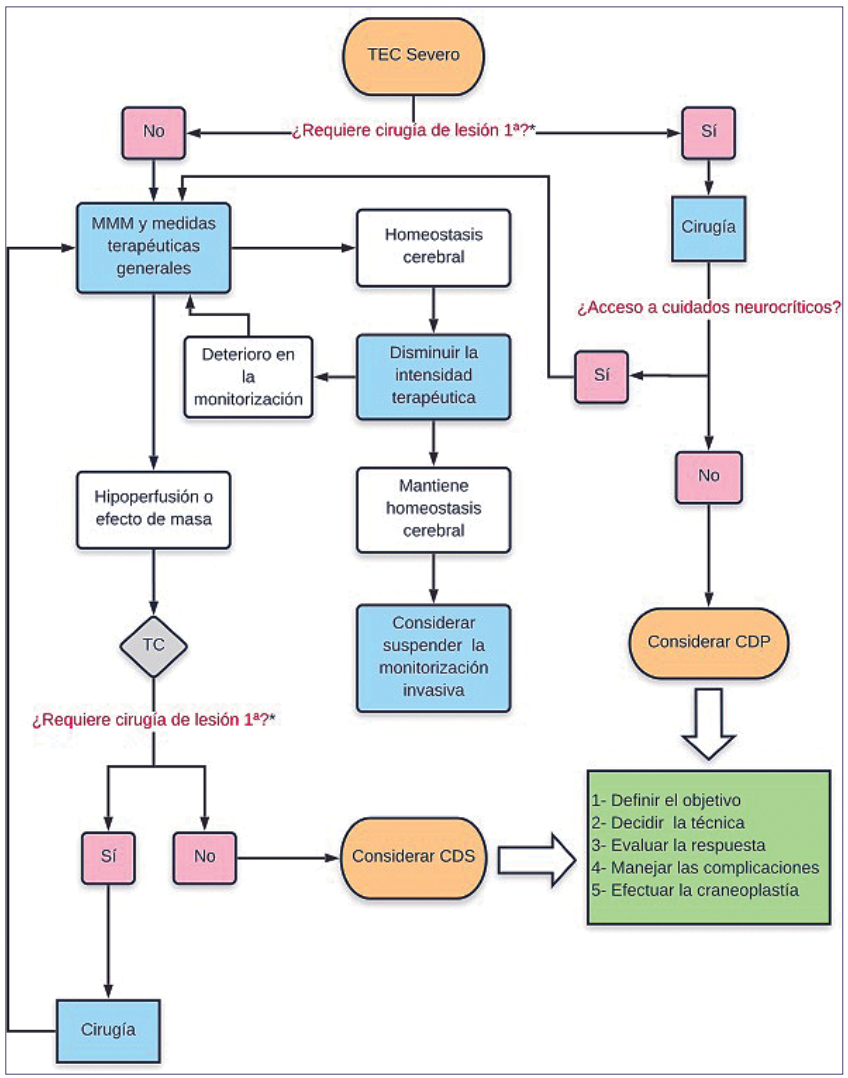

Figura 1. Proceso de toma de decisión para la indicación de una craniectomía descompresiva primaria y secundaria. Una vez decidida la craniectomía, los aspectos que deberían ser considerados para su ejecución se muestran secuencialmente en el rectángulo verde. MMM: monitorización multimodal, CDP: craniectomía descompresiva primaria, CDS: craniectomía descompresiva secundaria

\section{Técnica quirúrgica}

Siendo consecuentes con la definición, se podría afirmar que no existe una técnica única para realizar una CD. Bastaría sólo con cumplir el objetivo de aumentar el continente craneano lo suficiente como para disminuir los efectos deletéreos de la HTIC.

En la práctica, sin embargo, dado que lo que se busca es aumentar la complacencia sólo del compartimento supratentorial y que la posición del paciente y la sujeción de la cabeza durante la cirugía imponen algunas limitaciones prácticas, existen sólo dos grandes variantes técnicas de CD: bifrontal y frontotemporoparietal (también llamada hemicraniectomía).

1. Craniectomía descompresiva bifrontal: el paciente se posiciona en decúbito supino y, tras una incisión bicoronal, se puede lograr la remoción de la bóveda craneana hasta el piso de la fosa anterior y de ambas fosas medias. La durotomía se realiza típicamente siguiendo el contorno del área expuesta, pero ligeramente alejada $(1 \mathrm{~cm})$ del hueso y respetando el seno sagital superior (SSS) en su aspecto posterior. En el aspecto anterior, el SSS puede ser ligado y, en algunos casos, además se divide completamente la hoz del cerebro. Para cerrar, se realiza una duroplastía con injerto autólogo o heterólogo, o simplemente se cubre el cerebro expuesto con Surgicel ${ }^{5} y$, posteriormente, se 
aproximan la galea y la piel, procurando no disminuir la complacencia lograda 6 .

2. Hemicraniectomía: el paciente se posiciona en decúbito supino con la cabeza rotada hacia contralateral y, tras una incisión tipo colgajo o en "n", se puede lograr un remoción significativa de la bóveda craneana sobre los lóbulos parietal, frontal y temporal, llegando hasta el piso de la fosa anterior y de la fosa media ipsilateral. La durotomía típicamente se realiza siguiendo el contorno del área expuesta, pero ligeramente alejada del hueso $(1 \mathrm{~cm})$ y manteniendo un pedículo hacia la banda frontotemporal, aunque existen muchas otras variantes técnicas descritas, como las durotomías lineales múltiples ${ }^{8}$ o la durotomía estrellada. Para cerrar, se realiza una duroplastía con injerto autólogo o heterólogo, o simplemente se cubre el cerebro expuesto con Surgicel y, posteriormente, se aproximan la galea y la piel, procurando no disminuir la complacencia lograda.

En términos generales, el tipo de CD que se utiliza depende fundamentalmente de los hallazgos observados en la tomografía computada. En aquellos pacientes que presentan lesiones difusas, sin predominancia de algún hemisferio y, por lo tanto, sin desviación de línea media, existe una mayor tendencia a realizar CD bifrontales. Por contraparte, en aquellos pacientes con efecto de masa unilateral, se prefiere la hemicraniectomía.

Es importante aclarar que lo anterior es meramente una descripción general de ambas técnicas, pues existen muchas variantes descritas para cada una de las etapas de la cirugía. Entre estas variantes se encuentran: el tipo de incisión, el tamaño y localización de la área de cráneo removida, el tipo de durotomía, la realización o no de duroplastía, el tipo de duroplastía, la remoción del músculo temporal ${ }^{9}$ y la remoción del arco cigomático ${ }^{10}$. El conocimiento de estas distintas alternativas, con sus respectivas ventajas y desventajas, le otorga al equipo tratante una mayor capacidad para ajustar la técnica quirúrgica a las necesidades particulares de cada paciente y entender la respuesta a la cirugía y las potenciales complicaciones postoperatorias.

\section{Efectos de la craniectomía descompresiva}

La principal aprensión de quienes no son partidarios de utilizar la CD como herramienta terapéutica en el TEC severo, radica en que aún no somos capaces de entender realmente cómo ésta afecta los procesos fisiopatológicos subyacentes. De esta forma, si bien es un hecho aceptado (y respaldado por la evidencia $)^{11}$ que la CD disminuye la mortalidad, no está claro aún cuáles son sus consecuencias sobre la carga final de lesión cerebral secundaria y, por lo mismo, sobre el resultado funcional de los pacientes. Entre los principales efectos de la CD se encuentran (Figura 2):

\section{Disminución de la PIC}

El efecto inmediato de la CD, y que la mayoría de las veces es precisamente el efecto deseado, es la disminución de la PIC.

El descenso de la PIC durante la cirugía se produce en dos etapas. Un primer descenso se produce tras la remoción del cráneo. Esta disminución es significativa, pero generalmente insuficiente para lograr la normalización de la PIC, pues el cerebro queda contenido por la duramadre. Al hacer la durotomía, se produce un segundo gran descenso de la PIC, que puede en cierto modo titularse según la técnica de durotomía utilizada ${ }^{12}$. En definitiva, el descenso de la PIC depende de una herniación cerebral transcalvaria que, si bien es controlada, puede tener consecuencias negativas.

El gradiente de presión transcalvario que se genera durante el procedimiento debe tenerse presente en todo momento, pues podría desencadenar la progresión hemorrágica de contusiones cerebrales subyacentes y de hematomas extra-axiales contralaterales ${ }^{13}$.

Otra posible consecuencia adversa es el infarto del parénquima cerebral herniado, que se produce en algunos casos en que las arterias corticales quedan estranguladas contra la duramadre. El riesgo de que esto ocurra es directamente proporcional al volumen de parénquima herniado e inversamente proporcional al área de la durotomía. Sobre esta premisa se sustenta la recomendación, universalmente aceptada, de que el área de la craniectomía debe ser lo más amplia posible, buscando al menos $12 \times 15 \mathrm{~cm}$ de diámetro para una hemicraniectomía ${ }^{14}$.

\section{Alteraciones hemodinámicas}

A pesar de que el TEC no es una enfermedad, sino más bien un grupo de entidades nosológicas distintas que comparten su origen en el intercambio agudo de energía externa, la investigación nos ha mostrado que las alteraciones hemo-

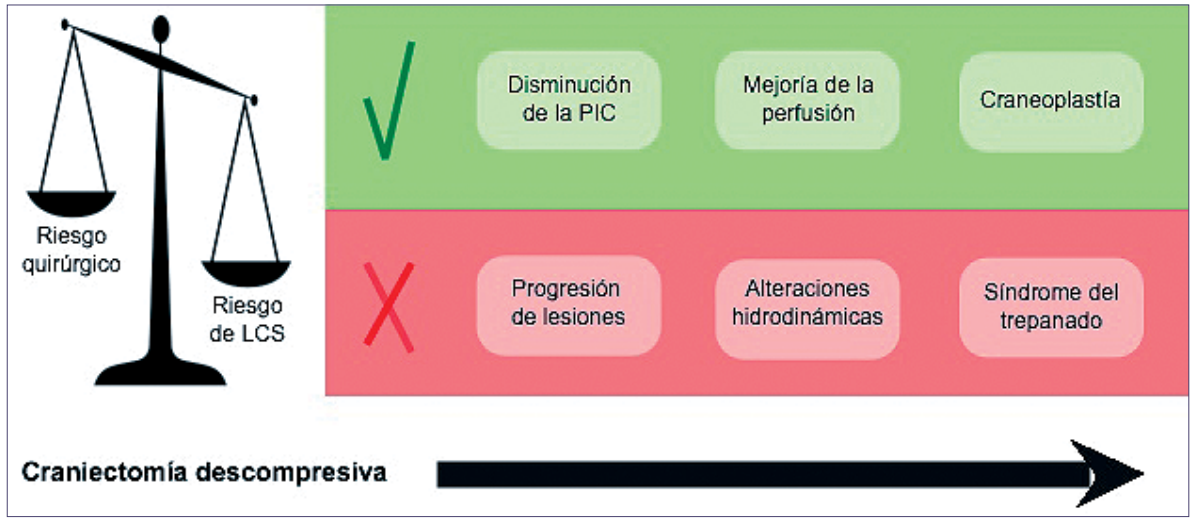

Figura 2. Evaluación de riesgo y consecuencias de la craniectomía descompresiva. Cuando el riesgo de LCS supera el riesgo quirúrgico, se debe considerar la CD. El efecto inmediato, cuando no hay progresión de lesiones, es la disminución de la PIC. En el período subagudo pueden presentarse las alteraciones hidrodinámicas $\mathrm{y}$, más tardíamente, el síndrome del trepanado. 
dinámicas que se producen en estos pacientes son bastante constantes $^{15}$. La capacidad de autorregulación cerebral se ve comprometida significativamente, el FSC aumenta en la etapa inicial y, posteriormente, disminuye. Los datos señalan, además, que el resultado funcional de los pacientes es peor en aquellos que evolucionan con alteraciones hemodinámicas más severas.

Las escasas líneas de investigación que abordan los efectos que tiene la CD sobre la hemodinamia cerebral, no permiten tener una idea clara al respecto. El hallazgo más constante pareciera ser la hiperemia regional del parénquima descomprimido ${ }^{16}$, con un consiguiente aumento de la oxigenación cerebral ${ }^{17}$. También se ha sugerido, sin embargo, que la CD podría mejorar la capacidad de autorregulación cerebral, produciéndose una disminución de la perfusión regional tras la cirugía ${ }^{18}$.

Teniendo en cuenta la evidencia presentada anteriormente, no es posible desligar la CD de los procesos fisiopatológicos que culminan en la LCS. Pero ¿en qué sentido podría alterarlos? La respuesta parece aún más elusiva cuando consideramos que la fisiopatología del TEC es extremadamente dinámica y la $\mathrm{CD}$, en cambio, como cualquier cirugía, es una terapia irreversible (al menos en la fase aguda) y muy poco dosificable.

\section{Alteraciones hidrodinámicas}

Las alteraciones hidrodinámicas que se producen en los pacientes con TEC severo, se hacen rápidamente evidentes con el seguimiento imagenológico que se realiza hoy en día a la mayoría de los pacientes. La hidrocefalia, los higromas y el edema cerebral, son algunos de los fenómenos que frecuentemente se observan en las tomografías computadas postoperatorias y que se podrían interpretar como la manifestación de una disfunción de la hidrodinamia intracraneana secundaria a la cirugía, pero cuyo origen aún no tiene una explicación sólida. Probablemente esto se deba a que es la fisiología hidrodinámica misma la que no entendemos adecuadamente $^{19}$. Explicar, por lo tanto, de qué forma la CD podría alterar la hidrodinamia intracranena desde un punto de vista mecanicista, pareciera por ahora estar fuera de nuestro alcance. En cualquier caso, la mera observación pareciera indicar que la incidencia, tanto de hidrocefalia como de higromas, es mayor en los pacientes descomprimidos y que esto, además, se asocia a peores resultados funcionales ${ }^{20}$.

\section{Evidencia y craniectomía descompresiva}

Bajo la concepción tradicional, en que la mejor evidencia disponible respecto al beneficio de una intervención/tratamiento proviene de los estudios clínicos aleatorizados de buena calidad metodológica, podríamos afirmar que, para la $C D$, la evidencia proviene fundamentalmente de 2 trabajos relativamnte recientes ${ }^{21}$ : DECRA trial ${ }^{22}$ y RESCUEicp ${ }^{23}$.

En ambos estudios, el objetivo principal fue evaluar el beneficio de la CDS utilizando el Glasgow Extended Outcome Scale (GOSE) a 6 meses. Las principales diferencias entre ambos trabajos, consisten en el criterio utilizado para definir HTIC refractaria, el tipo de pacientes incluidos y la técnica quirúrgica utilizada. En términos generales, el DECRA trial in- cluyó pacientes con menor carga de lesión cerebral primaria, definió HTIC con un umbral más bajo y utilizó una técnica quirúrgica estandarizada, mientras que el RESCUEicp no exigió una técnica quirúrgica predeterminada. DECRA trial no encontró diferencias significativas entre ambos grupos, mientras que RESCUEicp reportó una disminución considerable de la mortalidad en el grupo craniectomizado, acompañada de un aumento en el porcentaje de pacientes con discapacidad.

Estos resultados, ampliamente comentados y debatidos por los expertos, se presentan en opinión de los autores como evidencia de que la CDS, utilizada con los criterios propuestos por el RESCUEicp, disminuye categóricamente la mortalidad y podría incluso disminuir el riesgo de resultado funcional desfavorable de los pacientes, pero se requiere mayor investigación para precisar mejor cuáles son los pacientes que realmente se benefician de la intervención.

A partir en gran medida de esta evidencia, es que las recomendaciones actuales de la Brain Trauma Foundation respecto a CD son ${ }^{24}$ :

1. No se recomienda realizar $C D$ bifrontales utilizando los criterios del DECRA, para mejorar el GOSE a 6 meses.

2. Se recomienda que las hemicraniectomías realizadas sean de $12 \times 15 \mathrm{~cm}$ y no menores, para disminuir la mortalidad y mejorar los resultados funcionales.

Es interesante destacar que, pese a que no existe evidencia de que la CD mejore los resultados funcionales de los pacientes con TEC severo, la tendencia en los principales centros de referencia de neurotrauma, sigue siendo la de impulsar la investigación a entender en qué tipo de pacientes la CD podría ser beneficiosa. Para esto, connotados autores sugieren abandonar el paradigma de los $\mathrm{RCT}^{25}$ y volcar el esfuerzo científico a la producción de evidencia basada en estudios de efectividad comparativa (CER), planteando la problemática desde la perspectiva de la medicina de precisión. Un ejemplo de esto, es el desarrollo de recientes iniciativas colaborativas internacionales, como Center-TBI (https://www. center-tbi.eu).

Desde la mirada de la medicina de precisión, la falta de evidencia que respalde la CD se debe a que esta intervención se ha planteado siempre en el contexto de un protocolo, con umbrales preconcebidos y conductas estereotipadas, donde la cirugía se plantea como el último recurso tras múltiples tratamientos fallidos, con el objetivo es disminuir la PIC al mínimo posible. Esto se traduce, por ejemplo, en conductas como la de retirar la monitorización de PIC tras la cirugía, confiando en que la normalización de la PIC se mantendrá en el tiempo y, de no ser así, se entiende que se habría realizado ya el último esfuerzo terapéutico posible, por lo que se debería considerar fútil continuar el tratamiento.

Quizás el rol de la CD en el TEC severo sólo pueda definirse con claridad una vez que se abandone el paradigma actual del manejo de la PIC (escalonado, protocolizado), y se cambie por otro en que, tanto la toma de decisión como el tipo de cirugía propuesta, no se basen en un protocolo sino que en la interpretación fisiopatológica que surja del análisis de la monitorización multimodal del paciente individual. Así, por ejemplo, un paciente con un lóbulo temporal tumefacto y con signos precoces de herniación uncal en la pupilometría y la tomografía computada, podría beneficiarse de una craniec- 
tomía descompresiva dirigida específicamente a aumentar la complacencia de la fosa media, aun cuando la PIC y el resto de los parámetros de monitorización se encuentren en rango normal. De manera inversa, en cambio, un paciente con lesión cerebral difusa y una PIC media en $25 \mathrm{mmHg}$, pero con parámetros de monitorización del metabolismo cerebral en rango normal, podría no requerir escalar en la intensidad del tratamiento. Este cambio de paradigma implica, necesariamente, que la CD debe cumplir objetivos específicos, que deben ser planificados en conjunto por el neurointensivista y el neurocirujano.

\section{Cuidados postoperatorios}

La CD es una intervención que, generalmente, se realiza en las etapas iniciales de la evolución. Esto significa que el manejo del paciente con TEC severo está lejos de finalizar tras la cirugía. Los aspectos más relevantes a considerar durante el postoperatorio se abordan a continuación:

\section{Monitorización}

Los episodios de HTIC después de la CD, son una complicación reconocida y reportada en múltiples trabajos ${ }^{26}$. Si el objetivo de la cirugía era bajar la PIC, la monitorización de PIC intraoperatoria, y más aún la postoperatoria, para objetivar el resultado quirúrgico, resulta imprescindible y no se debe interrumpir hasta no tener claridad de que se ha superado la cima de la curva evolutiva de lesión cerebral.

\section{Complicaciones}

La mayoría de las complicaciones directamente atribuibles a la cirugía fueron mencionadas en el apartado sobre los efectos de la $C D$. Desde un punto de vista práctico, es útil agruparlas de acuerdo al momento del postoperatorio en el que más probablemente se presentan.

a. Inmediatas

Cirugía insuficiente: la "dosis" de descompresión no es suficiente y, por lo tanto, el objetivo de disminución de PIC no se logra.

Progresión de contusiones y hematomas: lesiones primarias que no requirieron remoción quirúrgica en un primer momento, pueden siempre aumentar de volumen por historia natural, pero esto además puede ser favorecido por el gradiente de presión transcalvario inducido por la CD.

b. Subagudas

- Alteraciones hidrodinámicas: la dilatación del sistema ventricular y la generación de colecciones extraaxiales tales como fístulas contenidas e higromas, son un problema que se genera con frecuencia tras la CD. Su presentación en general es insidiosa, pudiendo pasar desapercibida a la MMM invasiva y se asocian a un retraso en el proceso de neuro-rehabilitación. Por lo anterior, requieren un alto índice de sospecha, tratamiento precoz y prevención mediante la reparación precoz del defecto ${ }^{27}$.

c. Tardías

Complicaciones de la herida operatoria: las dehiscencias, las úlceras y la necrosis del extremo distal son complicaciones probablemente sub-reportadas, que se presentan en las primeras semanas del postoperatorio y que muchas veces son de difícil tratamiento ${ }^{28}$. Si bien los factores propios del paciente son determinantes (comorbilidades, calidad de los tejidos y su vascularización), su incidencia puede disminuirse en gran medida utilizando una buena técnica quirúrgica.

- Síndrome del trepanado: la presión subatmosférica intracraneana puede inducir una herniación paradójica, con un consecuente deterioro neurológico que sólo puede ser resuelto mediante la reparación del defecto.

El análisis de las características del paciente (epidemiológicas y clínicas), la técnica quirúrgica empleada y el tiempo transcurrido tras la cirugía, son fundamentales para la detección precoz y el tratamiento oportuno de estas complicaciones. La HTIC en el postoperatorio inmediato, por ejemplo, es más probablemente secundaria a una cirugía insuficiente o a la progresión hemorrágica de una lesión intracraneana, mientras que el deterioro neurológico a los pocos días de la cirugía podría deberse a un trastorno hidrodinámico.

\section{Reparación del defecto}

En los últimos años, la idea tradicional de esperar al menos 3 meses antes de realizar la reparación del defecto (craneoplastía), para disminuir el riesgo de infección del injerto, ha sido objetada por múltiples trabajos que muestran el beneficio de la craneoplastía en la rehabilitación neurológica de los pacientes, que incluso serían mayores cuando la craneoplastía se realiza precozmente $(<\text { a } 1 \text { mes })^{29}$. Pese a lo anterior, la evidencia al respecto sigue siendo controversial por lo que, si bien la realización precoz de la craneoplastía es aparentemente un ideal, los pacientes candidatos para este procedimiento deben ser seleccionados cuidadosamente.

\section{Comentario final y recomendaciones}

La CD es un método muy eficaz para disminuir la PIC y la mortalidad del TEC severo, sin embargo, dada la morbilidad asociada y el impacto controversial en el resultado funcional de los pacientes, su indicación debería considerar lo siguiente:

1. Indicar caso a caso.

2. Planificar en equipo.

3. Determinar objetivos específicos.

4. Evaluar el cumplimiento de los objetivos.

5. Mantener la monitorización multimodal postoperatoria.

6. Anticipar y tratar oportunamente las complicaciones.

7. Efectuar precozmente la craneoplastía.

\section{Referencias}

1. Le Roux P. Intracranial Pressure Monitoring and Management. En: Laskowitz D, editor. Translational Research in Traumatic Brain Injury. Boca Raton, FL: CRC press; 2016. p.365-378.

2. Kolias A, Kirkpatrick P, Hutchinson P. Decompressive craniectomy: past, present and future. Nat Rev Neurol. 2013;9(7):40515.

3. Kolias AG, Adams H, Timofeev I, Czosnyka M, Corteen EA, 
Pickard JD, Turner C, Gregson BA, Kirkpatrick PJ, Murray GD, Menon DK, Hutchinson PJ. Decompressive craniectomy following traumatic brain injury: developing the evidence base. Br J Neurosurg. 2016;30(2):246-50

4. Al-Jishi A, Saluja RS, Al-Jehani H, Lamoureux J, Maleki M, Marcoux J. Primary or secondary decompressive craniectomy: different indication and outcome. Can J Neurol Sci. 2011;38(4):612-20.

5. Güresir E, Vatter H, Schuss P, Oszvald A, Raabe A, Seifert V, Beck J. Rapid closure technique in decompressive craniectomy. J Neurosurg. 2011;114(4):954-60.

6. Timofeev I, Santarius T, Kolias AG, Hutchinson PJ. Decompressive craniectomy - operative technique and perioperative care. Adv Tech Stand Neurosurg. 2012;38:115-36.

7. Yang HS, Hyun D, Oh CH, Shim YS, Park H, Kim E. A Faster and Wider Skin Incision Technique for Decompressive Craniectomy: n-Shaped Incision for Decompressive Craniectomy. Korean J Neurotrauma. 2016;12(2):72-76.

8. Moringlane RB, Keric N, Freimann FB, Mielke D, Burger R, Duncker $D$ et al. Efficacy and safety of duotomy after decompressive hemicraniectomy in traumatic brain injury. Neurosurg Rev. 2017;40(4):655-661.

9. Yu SH, Kim BC, Choi JY, Lee JI, Cho WH, Choi HJ. Addition of Resection of Temporal Muscle and Fascia in Decompressive Craniectomy in the Treatment of Traumatic Brain Injury. Korean J Neurotrauma. 2016;12(2):84-88.

10. Martin AG, Abdullah JY, Jaafar A, Ghani AR, Rajion ZA, Abdullah JM. Addition of zygomatic arch resection in decompressive craniectomy. J Clin Neurosci. 2015;22(4):735-9.

11. Sahuquillo J, Dennis JA. Decompressive craniectomy for the treatment of high intracranial pressure in closed traumatic brain injury. Cochrane Database of Systematic Reviews 2019, Issue 12. Art. No.: CD003983.

12. Burger R, Duncker D, Uzma N, Rhode V. Decompressive craniotomy: durotomy instead of duroplasty to reduce prolonged ICP elevation. Acta Neurochir Suppl. 2008;102:93-7.

13. Kurland DB, Khaladj-Ghom A, Stokum JA, Carusillo B, Karimy JK, Gerzanich V et al. Complications Associated with Decompressive Craniectomy: A Systematic Review. Neurocrit Care. 2015;23(2):292-304.

14. Jiang JY, Xu W, Li WP, Xu WH, Zhang J, Bao YH et al. Efficacy of standard trauma craniectomy for refractory intracranial hypertension with severe traumatic brain injury: a multicenter, prospective, randomized controlled study. J Neurotrauma. 2005;22(6):623-628.

15. Donnelly J, Budohoski K, Smielewski P, Czosnyka M. Regulation of the cerebral circulation: bedside assessment and clinical implications. Crit Care. 2016;20(1):129.

16. Yamakami I, Yamamura A. Effects of decompressive craniectomy on regional cerebral blood flow in severe head trauma patients. Neurol Med Chir (Tokyo). 1993;33(9):616-20.

17. Lubillo ST, Parrilla DM, Blanco J, Morera J, Dominguez J, Belmonte F, López P, Molina I, Ruiz C, Clemente FJ, Godoy DA. Prognostic value of changes in brain tissue oxygen pressure before and after decompressive craniectomy following severe traumatic brain injury. J Neurosurg. 2018;128(5):1538-1546.

18. Amorim RL, Bor-Seng-Shu E, S Gattás G, Paiva W, de Andrade AF, Teixeira MJ. Decompressive craniectomy and cerebral blood flow regulation in head injured patients: a case studied by perfusion CT. J Neuroradiol. 2012;39(5):346-9.

19. Klarica M, Radoš M, Orešković D. The Movement of Cerebrospinal Fluid and Its Relationship with Substances Behavior in Cerebrospinal and Interstitial Fluid. Neuroscience. 2019;414:28-48.

20. Honeybul S, Ho KM. Incidence and risk factors for post-traumatic hydrocephalus following decompressive craniectomy for intractable intracranial hypertensiion and evacuation of mass lesions. J Neurotrauma. 2012;29(10):1872-8.

21. Kolias A, Viaroli E, Rubiano A, Adams H, Khan T, Gupta D et al. The Current Status of Decompressive Craniectomy in Traumatic Brain Injury. Curr Trauma Rep. 2018;4(4):326-332.

22. Cooper DJ, Rosenfeld JV, Murray L, Arabi YM, Davies AR, D'Urso $P$ et al. Decompressive craniectomy in diffuse traumatic brain injury. N Engl J Med. 2011;364(16):1493-502.

23. Hutchinson PJ, Kolias AG, Timofeev IS, Corteen EA, Czosnyka $\mathrm{M}$, Timothy $\mathrm{J}$ et al. Trial of Decompressive Craniectomy for Traumatic Intracranial Hypertension. N Engl J Med. 2016;375(12):1119-30.

24. Carney N, Totten AM, O'Reilly C, Ullman JS, Hawrlyuk GW, Bell $\mathrm{MJ}$ et al. Guidelines for the Management of Severe Traumatic Brain Injury, Fourth Edition. Neurosurgery. 2017;80(1):6-15.

25. Chesnut R. Craniectomy for Traumatic Intracranial Hypertension. N Engl J Med. 2016;375(24):2403.

26. Picetti E, Caspani M, laccarino C, Pastorello G, Salsi P, Viaroli E et al. Intracranial pressure monitoring after primary decompressive craniectomy in traumatic brain injury: a clinical study. Acta Neurochir (Wien). 2017;159(4):615-622.

27. Nalbach SV, Ropper AE, Dunn IF, Gormley WB. Craniectomyassociated Progressive Extra-Axial Collections with Treated Hydrocephalus (CAPECTH): redefining a common complication of decompressive craniectomy. J Clin Neurosci. 2012 Sep;19(9):1222-7.

28. Di Rienzo A, Pangrazi PP, Riccio M, Colasanti R, Ghetti I, lacoangeli M. Skin flap complications after decompressive craniectomy and cranioplasty: Proposal of classification and treatment options. Surg Neurol Int. 2016 Nov 9;7(Suppl 28):S737-S745.

29. Malcolm JG, Rindler RS, Chu JK, Choski F, Grossberg JA, Pradilla $G$ et al. Early Cranioplasty is Associated with Greater Neurological Improvement: A Systematic Review and MetaAnalysis. Neurosurgery. 2018;82(3):278-288. 